\title{
Mitochondria as biosynthetic factories for cancer proliferation
}

\author{
Christopher S Ahn ${ }^{1}$ and Christian M Metallo ${ }^{1,2^{*}}$
}

\begin{abstract}
Unchecked growth and proliferation is a hallmark of cancer, and numerous oncogenic mutations reprogram cellular metabolism to fuel these processes. As a central metabolic organelle, mitochondria execute critical biochemical functions for the synthesis of fundamental cellular components, including fatty acids, amino acids, and nucleotides. Despite the extensive interest in the glycolytic phenotype of many cancer cells, tumors contain fully functional mitochondria that support proliferation and survival. Furthermore, tumor cells commonly increase flux through one or more mitochondrial pathways, and pharmacological inhibition of mitochondrial metabolism is emerging as a potential therapeutic strategy in some cancers. Here, we review the biosynthetic roles of mitochondrial metabolism in tumors and highlight specific cancers where these processes are activated.
\end{abstract}

Keywords: Cancer, Mitochondria, Biosynthesis, Amino acids, Nucleotides, Anaplerosis, Lipogenesis

\section{Review}

Recent characterizations of metabolic enzymes as tumor suppressors and oncogene-driven metabolic reprogramming have reinvigorated interest in cancer metabolism. Although therapies targeting metabolic processes have long been a staple in cancer treatment (e.g. inhibition of folate metabolism via methotrexate), the focused therapeutic potential surrounding these findings have generated a renewed appreciation for Otto Warburg's work almost a century ago. Warburg observed that tumor cells ferment much of the glucose taken up during growth to lactate, thus using glycolysis as a major means of adenosine triphosphate (ATP) regeneration [1]. However, the observation of decreased respiration in cancer cells and idea that "the respiration of all cancer cells is damaged" belies the critical role of mitochondria in biosynthesis and cell survival [1]. On the contrary, functional mitochondria are present in all proliferative cells within our body (including all tumors), as they are responsible for converting the diverse nutrients available to cells into the fundamental building blocks required for cell growth. These organelles execute numerous functions in cancer cells to promote tumor growth and

\footnotetext{
*Correspondence: cmetallo@ucsd.edu

'Department of Bioengineering, University of California, San Diego, La Jolla, CA 92093, USA

${ }^{2}$ Moores Cancer Center, University of California, San Diego, La Jolla, CA 92093, USA
}

survival in response to stress. Here, we outline the critical biosynthetic functions served by mitochondria within tumors (Figure 1). Although many of these functions are similarly important in normal, proliferating cells, we have attempted to highlight potential points where mitochondrial metabolism may be therapeutically targeted to slow cancer growth. This review is organized by specific metabolic pathways or processes (i.e., glucose metabolism and lipogenesis, amino acid metabolism, and nucleotide biosynthesis). Tumors or cancer cell types where enzymes in each pathway have been specifically observed to by dysregulated are described within the text and summarized in Table 1.

\section{Glucose anaplerosis}

Glucose is the most widely available nutrient in our body; not surprisingly, most tumor cells consume this carbohydrate (or analogs) at high rates. This phenotype allows for detection and imaging of some cancers and metastatic lesions using the glucose analog 2-deoxy-2$\left[{ }^{18} \mathrm{~F}\right]$ fluoro-D-glucose (FDG), which accumulates in tumors (and some other tissues) and can be noninvasively observed when using positron emission tomography integrated with computed tomography (FDG-PET/CT) [2]. While FDG-PET/CET tracks cells with high glucose uptake and phosphorylation only, the use of isotope tracers, mass spectrometry (MS), and nuclear magnetic 


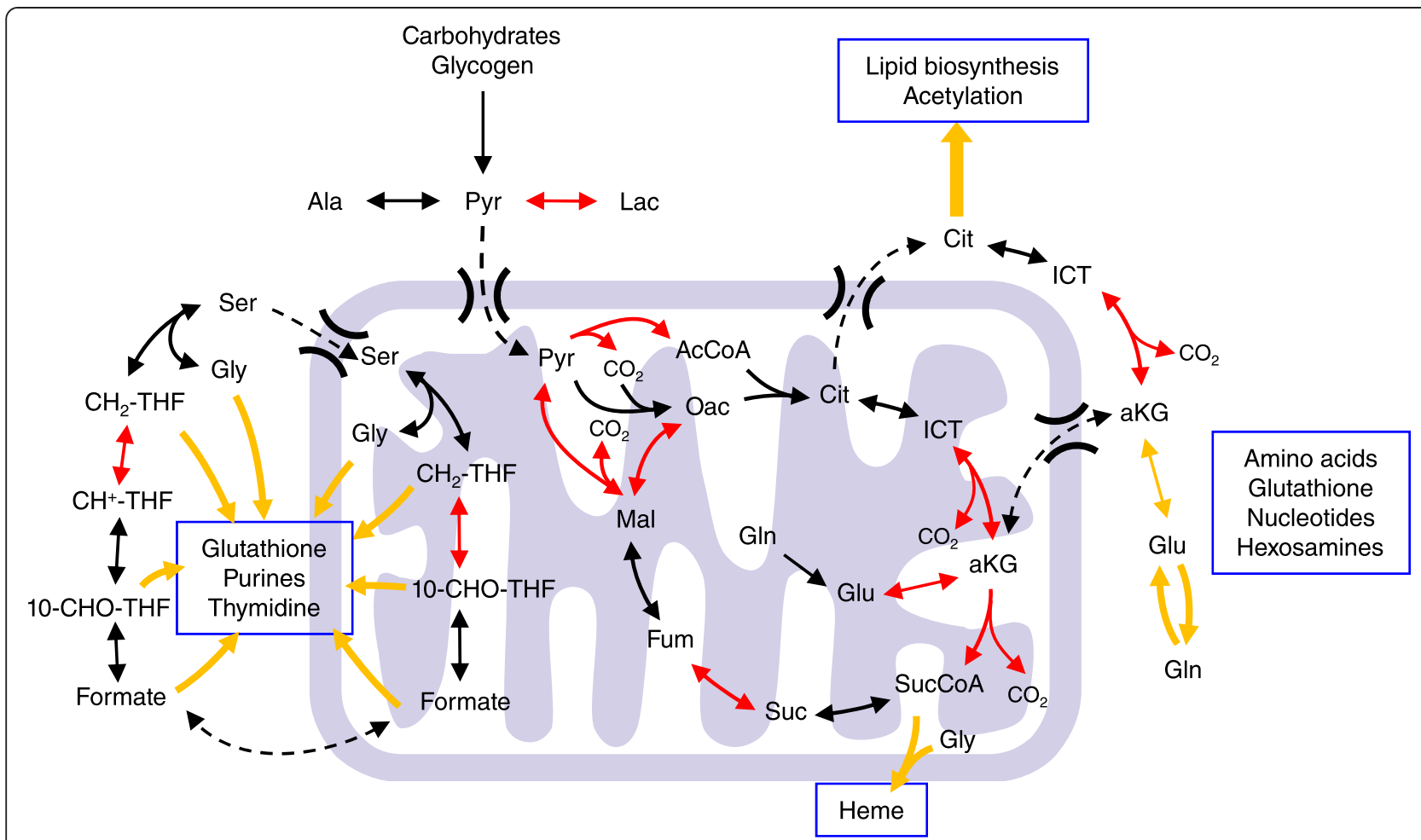

Figure 1 Biosynthetic nodes within mitochondria. Metabolic pathways within mitochondria that contribute to biosynthesis in cancer and other proliferating cells. TCA metabolism and FOCM enable cells to convert carbohydrates and amino acids to lipids, non-essential amino acids, nucleotides (including purines used for cofactor synthesis), glutathione, heme, and other cellular components. Critical biosynthetic routes are indicated by yellow arrows. Enzymatic reactions that are dependent on redox-sensitive cofactors are depicted in red.

resonance (NMR) have enabled researchers to more closely examine the fate of glucose within cancer cells [3-5]. Indeed, flux through glycolysis and lactate secretion remains a hallmark of many tumor cells, presumably to facilitate biosynthesis of ribose, purines (via serine and glycine), and lipid headgroups (via glycerol-3phosphate and serine). However, increasing evidence now indicates that cancer cells transport a significant portion of glucose-derived pyruvate into mitochondria where it serves as an anaplerotic substrate to replenish tricarboxylic acid (TCA) cycle intermediates used for biosynthesis. For example, conditions of limited glutamine availability or glutaminase (GS) suppression drive cancer cells to increasingly rely on glucose carbon flux through pyruvate carboxylase (PC) to maintain oxaloacetate (OAC) production and downstream TCA cycle activity [6]. Furthermore, NMR analysis of mice bearing three distinct human orthotopic tumors and infused with $\left[3,4-{ }^{13} \mathrm{C}_{2}\right]$ glucose indicated these glioblastoma lines used glucose as a mitochondrial anaplerotic substrate [7]. Although glutamine is one of the most abundant amino acids present in plasma, typical in vitro culture media used for cell line expansion contain relatively high concentrations $(2-4 \mathrm{mM})$ of glutamine. Thus, as tumor cells are increasingly analyzed prior to "adaptation/ selection" in vitro, we are beginning to better appreciate the importance of glucose-derived pyruvate as an anaplerotic substrate in tumors.

\section{Glucose oxidation and lipogenesis}

Beyond flux through PC or analogous reactions, the more predominant fate of mitochondrial pyruvate is oxidation by the pyruvate dehydrogenase (PDH) complex to form acetyl-coenzyme A (AcCoA). AcCoA is subsequently converted to citrate via condensation with OAC by citrate synthase. In turn, citrate is either converted to isocitrate in the TCA cycle or transported out of mitochondria and metabolized by ATP citrate lyase to yield cytosolic AcCoA, which is the substrate for de novo lipogenesis and acetylation. Mitochondrial activity within this pyruvate-citrate shuttle is therefore critical for the biosynthesis of fatty acids and cholesterol as well as protein acetylation. With some notable exceptions (e.g. hypoxia, discussed below), most cancer cells derive the majority of their lipogenic AcCoA from glucose-derived pyruvate through PDH [8-10]. Numerous oncogenic pathways stimulate glucose-derived carbon atom flux through the citrate shuttle to promote lipogenesis and TCA metabolism. Specific mutations in Kirsten rat sarcoma viral oncogene homolog (KRAS) stimulate flux of 
Table 1 Overview of mitochondrial biosynthetic enzymes important in cancer

\begin{tabular}{|c|c|c|c|c|c|c|c|c|c|c|c|c|}
\hline & \multicolumn{12}{|c|}{ Cancer type (according to primary site) } \\
\hline & $\begin{array}{l}\text { Blood, bone, } \\
\text { or lymph }\end{array}$ & Brain & Breast & Colon & Kidney & Liver & Lung & Stomach & Ovarian & Pancreas & Prostate & Skin \\
\hline \multicolumn{13}{|c|}{ TCA cycle, anaplerosis, and AcCoA metabolism } \\
\hline Pyruvate carboxylase & & • & • & • & • & • & • & & & • & & • \\
\hline Pyruvate dehydrogenase complex & • & - & • & • & - & & • & & & • & & • \\
\hline $\begin{array}{l}\text { Isocitrate dehydrogenase (activity } \\
\text { or mutation) }\end{array}$ & • & • & • & • & • & • & • & & & • & & • \\
\hline Succinate dehydrogenase (mutation) & & & & & - & & & & & & & \\
\hline Fumarate hydratase (mutation) & & & & & • & & & & & & & \\
\hline $\begin{array}{l}\text { Glutaminase and/or glutamate } \\
\text { dehydrogenase }\end{array}$ & • & • & • & • & • & - & • & & & $\cdot$ & & • \\
\hline Glutamine synthetase & & - & - & & & - & & & & & & \\
\hline \multicolumn{13}{|l|}{ Amino acid metabolism } \\
\hline Pyrroline-5-carboxylate reductase & • & - & • & • & • & & • & & • & • & • & • \\
\hline Proline oxidase & • & • & • & • & • & • & • & • & • & • & • & - \\
\hline Aspartate transaminase & & & • & & & • & & & & • & & \\
\hline Alanine transaminase & & & & - & & • & • & • & & • & & - \\
\hline \multicolumn{13}{|l|}{ Nucleotide biosynthesis } \\
\hline Dihydroorotate dehydrogenase & • & • & • & • & • & & - & & & & • & - \\
\hline $\begin{array}{l}\text { Methylenetetrahydrofolate } \\
\text { dehydrogenase }\end{array}$ & $\cdot$ & • & • & - & & - & • & • & • & $\cdot$ & $\cdot$ & • \\
\hline
\end{tabular}

Cancers in which three or more mitochondrial enzymes have been studied and found to be differentially regulated (or mutated, as indicated) in cancers vs. control groups are included. Dysregulation of each enzyme was demonstrated in clinical tumors samples, animal models, or cell lines at the levels of genes, mRNA, protein, metabolites, and/or flux.

glucose through PDH to generate fatty acids [11,12]. Alternatively, active Akt promotes glucose-mediated fatty acid synthesis downstream of PDH [8,13]. This Aktdependent lipogenesis occurs by activation of mammalian target of rapamycin complex 1 (mTORC1) and sterol regulatory element-binding protein 1 (SREBP1), which are key regulators of cellular growth and lipid homeostasis, respectively [14]. Interestingly, SREBPs have also been shown to coordinate lipid and protein biosynthesis as well as protect cancer cells from saturated fatty acid-induced lipotoxicity $[15,16]$. On the other hand, inactivation of mTORC1 reduces mitochondrial fluxes that supply the citrate and AcCoA which fuel these pathways [17,18]. Finally, overexpression of the HER2 oncogene or epidermal growth factor (EGF) stimulation both activate MEK/ERK signaling to suppress the inhibitory PDH kinase 4 (PDK4) and maintain glucose oxidation in mammary epithelial cells [19].

These above concepts and results contrast the established role of PDH kinase 1 (PDK1) in supporting tumor growth downstream of hypoxia-inducible factor (HIF) signaling by suppressing PDH activity [20-22]. Indeed, inhibition of PDK1 activity using dichloroacetate (DCA) forces glucose oxidation under hypoxic conditions [9] and inhibits the growth of xenograft tumors [23]. Limited mitochondrial glucose metabolism due to hypoxic or pseudohypoxic stabilization of HIFs is a hallmark of some renal carcinomas [9,24,25], and normalization of HIF levels (thus increasing glucose oxidation) in such cells abrogates tumor formation in xenografts [26]. Suppression of PDK1 to activate PDH flux also contributes to BRAF(V600E)-induced oncogene senescence [27], further suggesting that limiting glucose oxidation is important for tumor growth. Furthermore, some tumors downregulate expression of the mitochondrial pyruvate carrier (MPC), and acute inhibition of the MPC in cancer cells significantly decreases glucose oxidation but has no effect on growth or respiration [28-30]. Tumor cells are clearly able to compensate for this lack of glucose-mediated biosynthesis under these conditions through extramitochondrial pathways, scavenging acetate [31,32], unsaturated lipids [8,33], or proteins [34] when required. Therefore, the relative importance of glucose-driven biosynthesis through mitochondrial pathways may be tumor specific. Alternatively, there may be a particular level of glucose flux into mitochondria that supports biosynthesis while limiting oxidative TCA metabolism and potentially deleterious byproducts (e.g. reactive oxygen species; ROS). Further mechanistic studies are required to characterize the mechanisms through which cancer cells balance mitochondrial energetic (catabolic) and biosynthetic (anabolic) metabolism. 


\section{Amino acid metabolism}

In addition to carbohydrates, amino acids are critical substrates fueling mitochondrial metabolism and the biosynthesis of proteins, lipids, and other molecules. Of particular interest in cancer are key mitochondrial enzymes in the metabolism of glutamine, glutamate, proline, aspartate, and alanine (Figure 2). Glutamine is one of the most critical nutrients required for cell proliferation, as the amido nitrogen of this amino acid is the obligate substrate for hexosamine and nucleotide biosynthesis in the cytosol. Furthermore, the carbon backbone of glutamine is an important anaplerotic substrate fueling TCA cycle metabolism (Figure 1). Upon conversion to glutamate via glutaminase (GLS) activity, N-acetyl-glucosamine production, or nucleotide biosynthesis, glutamine carbons enter the TCA cycle as alpha-ketoglutarate (aKG) downstream of glutamate dehydrogenase (GDH) or transaminase activity $[35,36]$. The GLS (rather than GLS2) isoform is commonly expressed in tumors and is regulated downstream of the $M Y C$ oncogene [37]. Pharmacological inhibition of GLS is being investigated as a potential means of therapy for a number of different tumor types [38-40]. Indeed, GLS facilitates oxidative glutaminolytic flux in tumor cells derived from gliomas, lymphomas, breast cancers, prostate cancers, pancreatic cancers, and melanomas [38,40-44]. Recent flux studies in tumor cells bearing isocitrate dehydrogenase 1 (IDH1) mutations indicate that these cells may be particularly dependent upon glutamine to fuel oxidative mitochondrial metabolism and thus may be responsive to inhibition of GLS or respiration [39,45]. GLS-derived glutamate is also important for glutathione synthesis, which is abundant at $\mathrm{mM}$ levels in cells and plays an important role in redox homeostasis and tumor cell survival in response to oxidative stress [46].

\section{Glutaminolysis and reductive carboxylation}

Glutamine carbon can also fuel AcCoA generation for lipid biosynthesis when metabolized by malic enzymes (MEs) through glutaminolysis or alternatively via reductive carboxylation. The former pathway, by which glutamine-derived malate is converted to pyruvate and subsequently lactate or AcCoA, is active in some tumor cells that express high levels of cytosolic ME1 or the other mitochondrial isozymes $M E 2$ and $M E 3$ [44,47].



Figure 2 Coordination of carbon and nitrogen metabolism across amino acids. Glutamate and aKG are key substrates in numerous transamination reactions and can also serve as precursors for glutamine, proline, and the TCA cycle. Mitochondrial enzymes catalyzing these reactions are highlighted in blue, and TCA cycle intermediates are highlighted in orange (pyruvate enters the TCA cycle as acetyl-CoA or oxaloacetate). 
Leukemic cells under hypoxia have been observed to employ this pathway for biosynthesis and ATP regeneration [38]. Glutaminolysis is also specifically activated in proliferating cells upon inhibition of MPC function, which may contribute to the sensitivity of cancer cells to inhibitors of glutamine metabolism $[29,30]$.

In contrast to the oxidative glutaminolytic pathway, reductive carboxylation involves the "reverse" or reductive activity of $\mathrm{NADP}^{+}$-dependent IDHs to generate isocitrate and citrate from aKG, a pathway which becomes important in cells proliferating in hypoxic microenvironments or those with dysfunctional mitochondria [9,25,42,48]. HIFs stabilized by low oxygen levels or loss of the von Hippel Lindau tumor suppressor reduce PDH activity $[20,21]$, leading cells to use alternate substrates for AcCoA generation such as glutamine or aKG [24]. In this manner, mass action and/or altered mitochondrial redox states induce proliferating cells to reductively metabolize aKG via $\mathrm{NADP}^{+}$-dependent IDHs and subsequently generate isocitrate and ultimately AcCoA [49]. Evidence also suggests that aKG-dehydrogenase $(O G D H)$ and nicotinamide nucleotide transhydrogenase (NNT) expression are both required for activation of this pathway [48,50]. Indeed, hypoxic cells maintain and even upregulate oxidative glutamine metabolism in mitochondria despite the observed increase in reductive carboxylation activity [38,45,51]. Thus, some mitochondrial functions are required to allow conversion of glutamine to AcCoA through this pathway. On the other hand, cells with heterozygous mutations in IDH1 are specifically compromised in their ability to use reductive carboxylation for fatty acid synthesis [45], suggesting that the cytosolic isozyme catalyzes reductive carboxylation. While the specific contributions and functions of mitochondrial IDH2 and cytosolic IDH1 in this pathway must be definitively characterized (both in vitro and in vivo), increased exchange of aKG and isocitrate/citrate occurs in the context of perturbed redox states when fatty acid biosynthesis is maintained, a common occurrence in the tumor microenvironment. Ultimately, this pathway may effectively allow cancer cells to maintain biosynthesis, transfer reducing equivalents between compartments, or both to support growth and survival in hypoxic microenvironments.

\section{Glutamine synthesis}

Many amino acids are not extremely abundant in plasma or the tumor microenvironment and therefore must be synthesized de novo. Mitochondrial metabolism plays a definitive role in the production of many non-essential amino acids and their further utilization in biosynthetic pathways. Although glutamine is relatively abundant in plasma, de novo glutamine synthesis in the liver and surrounding tissues is likely critical for tumor cell growth. Glutamine biosynthesis requires a supply of aKG from mitochondrial metabolism to generate glutamate (a critical precursor for most non-essential amino acids) and subsequently glutamine via glutamine synthetase (GS). De novo glutamine biosynthesis in tumors has been detected in vivo using infusions of $\left[{ }^{13} \mathrm{C}\right]$ glucose into mice bearing human glioblastoma orthotopic tumors [7]. Furthermore, some breast epithelial cells can mediate glutamine independence via expression of GS [52]. Finally, glutamine as well as other amino acids may be scavenged via protein catabolism when it is not available in sufficient quantities [34].

\section{Proline metabolism}

Mitochondrial proline metabolism and synthesis are critically important for tumor cells, at least in part due to the unique, modifiable chemical properties it provides to proteins. Proline is synthesized from glutamine or ureacycle-derived ornithine via the intermediate pyrroline-5carboxylate (P5C). P5C is then converted to proline via the $\mathrm{NAD}(\mathrm{P}) \mathrm{H}$-dependent enzyme pyrroline-5-carboxylate reductase (PYCR), which exists in three isoforms: PYCR1, PYCR2, and PYCRL (Figure 2). Mitochondrial PYCR1 and PYCR2 are upregulated in multiple types of cancer, including prostate, lymphoma, and others $[41,53,54]$. Overexpression of $c-M y c$ in P493 human Burkitt lymphoma and PC3 human prostate cancer induced an upregulation of PYCR1 expression as well as the P5C biosynthetic enzyme delta-1-pyrroline-5-carboxylate synthase (P5CS), resulting in higher levels of intracellular proline [41]. In line with this observation, expression of both PYCR1 and PYCR2 was increased in a panel of melanoma cell lines but was undetectable in normal melanocytes [53]. Furthermore, a recent large-scale comparative analysis of published mRNA microarray datasets found that PYCR1 was one of the most commonly overexpressed metabolic enzyme genes in comparison to normal tissue among the 19 represented cancer types [54]. Although the functional advantages provided to cancer cells by modulating proline metabolism are not completely clear, the importance of proline in extracellular matrix proteins (e.g. collagen) could play a role in tumorigenesis. Alternatively, interconversions of proline and $\mathrm{P} 5 \mathrm{C}$ in the cytosol and mitochondria have been proposed as a means of transferring reducing equivalents between these compartments [55], though more detailed functional analyses are required to elucidate how proline metabolism contributes to cancer progression.

Downregulation of proline catabolism is complementary to its biosynthesis and commonly observed in a number of tumor types. The first step of this process is catalyzed in the mitochondria by proline oxidase (POX), and the expression of this enzyme is markedly reduced in many cancers compared to normal tissue from the same patient [56]. POX expression is induced by the 


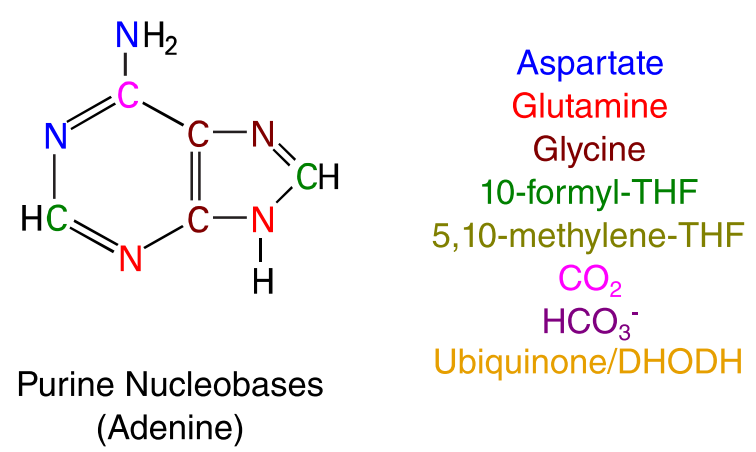<smiles>Cc1c[nH]c(=O)[nH]c1=O</smiles>

Pyrimidine Nucleobases (Thymine)

Figure 3 Biosynthetic sources for purine and pyrimidine synthesis. Sources and fates of nitrogen, carbon, and oxygen atoms are colored as indicated. Italicized metabolites can be sourced from the mitochondria or cytosol. The double bond formed by the action of DHODH/ubiquinone is also indicated.

tumor suppressor p53, and ectopic expression of $P O X$ in DLD-1 colon cancer cells induces cell cycle arrest and reduces tumor burden in xenograft models [56]. Furthermore, $P O X$ expression is inhibited by MYC via miR-23b* in lymphoma, renal, and prostate cancers [41,57]. The widespread repression of POX in cancer indicates that this enzyme may act as a tumor suppressor; however, the specific mechanisms through which POX deficiency promotes tumorigenesis are not yet clear.

\section{Aspartate and asparagine metabolism}

Aspartate can be generated from the TCA intermediate oxaloacetate by glutamate-mediated transaminase activity (Figure 2); thus, the biosynthesis of aspartate and downstream metabolites is intimately tied to mitochondrial activity. Aspartate transaminases (GOT1, cytosolic; GOT2, mitochondrial), which bidirectionally convert aspartate and aKG to OAC and glutamate, are important for the growth of human pancreatic adenocarcinoma (PDAC) [43]. Oncogenic KRAS, the most common mutation in PDAC, redirects glutamine metabolism toward aspartate production in a number of settings $[11,43,58]$. This metabolic reprogramming is thought to facilitate regeneration of NADPH for reductive biosynthesis and redox homeostasis as well as $\mathrm{NAD}^{+}$for maintaining glycolysis [43]. Ablation of oncogenic KRAS in a mouse model of pancreatic cancer markedly reduced tumor size and also revealed a subpopulation of surviving tumor cells which did not express KRAS. These surviving cells relied heavily on oxidative phosphorylation and were sensitive to oligomycin treatment, providing evidence that inhibition of mitochondrial function may effectively target cells that survive after suppression of oncogenic KRAS signaling [59]. Additionally, aspartate and glutamine are the precursors for asparagine, which is synthesized in the cytosol by asparagine synthetase (ASNS). ASNS expression is required for the survival of cultured glioma and neuroblastoma cell lines, and supplementation of exogenous asparagine can prevent apoptosis induced by glutamine withdrawal, in part, by modulating ER stress [60]. Expression of ASNS is also correlated with drug resistance in childhood acute lymphoblastic leukemia (cALL) and some forms of acute myeloblastic leukemia (AML), which are typically deficient in their ability to synthesize asparagine de novo $[61,62]$. Finally, aspartate is a key initiator of pyrimidine synthesis and donates nitrogen for purine synthesis via adenylosuccinate synthetase (Figure 3), further highlighting the role of mitochondrial aspartate metabolism in tumor cell biosynthesis.

\section{Alanine and BCAA metabolism}

Alanine production via alanine transaminases (GPT1, cytosolic; GPT2, mitochondrial), which transfer an amino group between glutamate and pyruvate to yield alanine and aKG, not only provide proteinogenic alanine but also aKG for TCA cycle activity (Figure 2). Maintenance of glutamine anaplerosis and catabolism in cancer cells via increased GPT2 activity is essential for oncogenic KRAS-induced anchorage independent growth, as demonstrated by knockdown of GPT2 expression in HCT116 colon cancer cells [35]. GPT activity may also facilitate disposal of excess nitrogen (such as that derived from glutamine) via alanine secretion [63]. Indeed, secretion of alanine is higher in melanoma cell lines compared to normal melanocytes and is quite significant in human colon carcinoma tumors $[35,64]$.

Finally, the branched chain amino acids (BCAAs) valine, leucine, and isoleucine are also highly metabolized by transaminases in both the cytosol (via BCAT1) and mitochondria (via BCAT2) (Figure 2) [65]. While cytosolic BCAT1 metabolism has been implicated in gliomas with wild-type IDH1 [66], how BCAA catabolism contributes to cancer progression remains unclear. Ultimately, by coordinating cellular bioenergetics and biosynthesis through the TCA cycle, amino acid metabolism plays a critical role in tumor growth and survival. 


\section{Nucleotide biosynthesis}

In addition to amino acid and lipid biosynthesis, nucleotide production is highly dependent upon mitochondrial metabolism and associated intermediates. While the ribose moiety of nucleotides is exclusively generated in the cytosol, many components that contribute to both pyrimidine and purine bases are derived directly or indirectly from mitochondria (Figure 3). Pyrimidine ring synthesis requires glutamine and aspartate, which can be supplied by mitochondrial pathways as noted above. Pyrimidine synthesis also requires the activity of dihydroorotate dehydrogenase (DHODH), a mitochondrial enzyme that converts dihydroorotate to orotate coupled with the reduction of ubiquinone to ubiquinol. Importantly, oxidation of ubiquinol in the electron transport chain (ETC) is necessary to maintain an adequate supply of ubiquinone for DHODH activity. In fact, uridine must be supplemented to culture media to allow proliferation of $\rho^{0}$ cells (i.e., cells lacking functional mitochondrial DNA) and other cell lines with genetic modifications that compromise respiration $[45,67]$. Thus, DHODH links cellular respiration and pyrimidine synthesis. Elevated $D H O D H$ expression and increased activity have been observed in multiple types of cancers (Table 1) [68-71]. Inhibition of DHODH in human melanoma decreases growth both in vitro and in murine xenografts [70]. Doxorubicin, a common chemotherapeutic, induces a decrease in $\mathrm{DHODH}$ expression and acts synergistically with tumor necrosis factor-related apoptosisinducing ligand (TRAIL) to selectively kill tumor cells [68]. DHODH is also suppressed by miR-502, which is expressed at significantly lower levels in human colon tumors relative to normal tissue [71]. Finally, suppression of DHODH also impairs the function of complex III in the ETC, causing accumulation of p53 and induction of apoptosis, which further relates mitochondrial respiration to cancer growth and survival [72].

Purine nucleotide synthesis requires nitrogen from aspartate and glutamate as well as glycine and formate for backbone synthesis (Figure 3). While enzymes involved in glycine and formate synthesis are present in both the cytosol and mitochondria, increasing evidence suggests that the formate (and potentially glycine) fueling this pathway is primarily derived from mitochondrial metabolism. Formate is incorporated into purines via 10-formyl-tetrahydrofolate (10-CHO-THF) and thymidine via 5,10-methylene-THF. These substrates can be generated in both the cytosol and mitochondria via serine hydroxymethyltransferase (SHMT), methylenetetrahydrofolate dehydrogenase (MTHFD), and downstream reactions in folate-mediated one carbon metabolism (FOCM) [73]. We recently developed a system for quantifying the contribution of different substrates to the mitochondrial and cytosolic NADPH pools using $\left[{ }^{2} \mathrm{H}\right]$ tracing and inducible expression of mutants IDH1 and IDH2 [74].
Application of $\left[{ }^{2} \mathrm{H}\right]$-labeled serine, glycine, and glucose tracers to non-small cell lung cancer cells indicated that serine flux through SHMT2 and MTHFD2(L) operates primarily in the oxidative direction to produce mitochondrial $\mathrm{NAD}(\mathrm{P}) \mathrm{H}$ in these cancer cells [74]. Additional evidence by others supports the concept that mitochondrial FOCM is an important contributor of reducing equivalents and one carbon intermediates for nucleotide biosynthesis $[75,76]$. While the cytosolic pathway may independently contribute to nucleotide biosynthesis [77], our results correlate with the recent demonstration that MTHFD2 expression is commonly elevated in many cancers and associated with poor survival in breast cancer patients [54].

\section{Conclusions}

Mitochondria operate as both engine and factory in eukaryotes, coordinating cellular energy production and the availability of fundamental building blocks that are required for cell proliferation. Cancer cells must therefore balance their relative bioenergetic and biosynthetic needs to grow, proliferate, and survive within the physical constraints of energy and mass conservation. In contrast to quiescent cells, which predominantly use oxidative mitochondrial metabolism to produce ATP and uptake glucose at much lower rates than proliferating cells, tumor cells exhibit increased glycolytic rates to provide an elevated flux of substrate for biosynthetic pathways, including those executed within mitochondria. Given these higher rates of nutrient utilization, metabolic flux through mitochondrial pathways and the associated ROS production can often be higher in cancer cells. Not surprisingly, activation of cellular antioxidant response pathways is commonly observed in cancer or subpopulations of cells within tumors [46,78]. Cellular compartmentalization affords a degree of protection from such damaging side products of metabolism, and methods which are able to deconvolute the relative contributions of each cellular compartment (e.g. mitochondria, cytosol, peroxisome, etc.) to cancer metabolism will be crucial to more completely understand the metabolism of cancer cells in the future [74,79]. Ultimately, while mitochondrial dysregulation is widely considered to be a hallmark of cancer, numerous mitochondrial functions remain critical for tumor growth and are emerging as clinical targets.

Following this point, it comes as no surprise that mitochondrial metabolism is highly active in virtually all tumors (i.e., cancer cells, stroma, or both), and investigators have begun targeting these pathways to explore potential efficacy. Indeed, some evidence suggests that biguanides such as metformin or phenformin may limit tumor incidence and burden in humans and animals $[80,81]$. These effects are presumably due, at least in 
part, to complex I inhibition of the ETC, which significantly perturbs mitochondrial function $[82,83]$. However, more insights are needed into the mechanisms of these compounds in patients to determine the therapeutic potential of targeting this and other components of mitochondria. In developing new therapies that target cancer metabolism, researchers will face challenges similar to those that are relevant for many established chemotherapies since deleterious effects on normal proliferating cells that also depend on mitochondrial metabolism (and aerobic glycolysis) are likely to arise.

As we acquire a more detailed picture of how specific genetic modifications in a patient's tumor correlate with its metabolic profile, opportunities for designing targeted or combinatorial therapies will become increasingly apparent. Cancer therapies that address tumorspecific mitochondrial dysregulation and dysfunction may be particularly effective. For example, some cancer cells harbor mutations in TCA enzymes (e.g., FH, SDH, IDH2) or regulatory proteins that control mitophagy (i.e., LKB1) [84]. Such tumors may be compromised with respect to some aspects of mitochondrial biosynthesis and dependent on alternate pathways for growth and/or survival such that synthetically lethal targets emerge. Ultimately, such strategies will require clinicians and researchers to coordinate metabolic, biochemical, and genetic information in the design of therapeutic strategies.

\begin{abstract}
Abbreviations
FDG: 2-deoxy-2-[ ${ }^{18}$ F]fluoro-D-glucose; $\mathrm{CH}_{2}$-THF: 5,10-methylene tetrahydrofolate; 10-CHO-THF: 10-formyl-tetrahydrofolate; ATP: adenosine triphosphate; GOT: aspartate transaminases; ASNS: asparagine synthetase; AML: acute myeloblastic leukemia; AcCoA: acetyl-coenzyme A; Ala: alanine; GPT: alanine transaminases; aKG: alpha-ketoglutarate; BCAT: branched chain aminotransferase; Cit: citrate; CALL: childhood acute lymphoblastic leukemia; P5CS: delta-1-pyrroline-5-carboxylate synthase; DHODH: dihydroorotate dehydrogenase; ETC: electron transport chain; EGF: epidermal growth factor; FOCM: folate-mediated one carbon metabolism; Fum: fumarate; FH: fumarate hydratase; GIn: glutamine; Glu: glutamate; Gly: glycine; GS: glutamine synthetase; GLS: glutaminase; GDH: glutamate dehydrogenase; HOTs: human orthotopic tumors; HIF: hypoxia inducible factor; ICT: isocitrate; IDH: isocitrate dehydrogenase; Lac: lactate; LKB1: liver kinase B1; MS: mass spectrometry; mTORC1: mammalian target of rapamycin complex 1; ME: malic enzymes; Mal: malate; MTHFD: methylenetetrahydrofolate dehydrogenase; $\mathrm{NADH}$ : nicotinamide adenine dinucleotide, reduced; NADPH: nicotinamide adenine dinucleotide phosphate, reduced; NNT: nicotinamide nucleotide transhydrogenase; NMR: nuclear magnetic resonance; OAC: oxaloacetate; PET/CT: positron emission tomography integrated with computed tomography; PDAC: pancreatic adenocarcinoma; Pyr: pyruvate; $\mathrm{PDH}$ : pyruvate dehydrogenase; PC: pyruvate carboxylase; PDK: PDH kinase; P5C: pyrroline5-carboxylate; PYCR: pyrroline-5-carboxylate reductase; POX: proline oxidase; ROS: reactive oxygen species; Ser: serine; SHMT: serine hydroxymethyltransferase; SREBP1: sterol regulatory element binding protein 1; Suc: succinate; SDH: succinate dehydrogenase; TCA: tricarboxylic acid; TRAIL: tumor necrosis factor-related apoptosis-inducing ligand.
\end{abstract}

\section{Competing interests}

The authors declare that they have no competing interests.

\section{Authors' contributions}

CSA and CMM discussed and wrote the manuscript. Both authors read and approved the final manuscript.

\section{Authors' information}

CSA is a Bioengineering Ph.D. student at the University of California, San Diego. CMM is an assistant professor in the Jacobs School of Engineering, Department of Bioengineering at the University of California, San Diego.

\section{Acknowledgements}

The authors acknowledge helpful comments from members of the Metallo laboratory. We apologize to those researchers whose work we were unable to cite. This work is supported by NIH grants 1R01CA188652 and

1R01CA132847, DOD grant W81XWH-13-1-0105, and a Searle Scholar Award.

Received: 29 September 2014 Accepted: 2 January 2015

Published online: 25 January 2015

\section{References}

1. Warburg O. On the origin of cancer cells. Science. 1956;123:309-14.

2. Farwell MD, Pryma DA, Mankoff DA. PET/CT imaging in cancer: current applications and future directions. Cancer. 2014;120:3433-45.

3. Maher EA, Marin-Valencia I, Bachoo RM, Mashimo T, Raisanen J, Hatanpaa KJ, et al. Metabolism of [U-13 C]glucose in human brain tumors in vivo. NMR Biomed. 2012;25:1234-44.

4. Richardson AD, Yang C, Osterman A, Smith JW. Central carbon metabolism in the progression of mammary carcinoma. Breast Cancer Res Treat. 2008;110:297-307.

5. Lane AN, Fan TW, Bousamra 2nd M, Higashi RM, Yan J, Miller DM. Stable isotope-resolved metabolomics (SIRM) in cancer research with clinical application to nonsmall cell lung cancer. OMICS. 2011;15:173-82.

6. Cheng T, Sudderth J, Yang C, Mullen AR, Jin ES, Mates JM, et al. Pyruvate carboxylase is required for glutamine-independent growth of tumor cells. Proc Natl Acad Sci U S A. 2011;108:8674-9.

7. Marin-Valencia I, Yang C, Mashimo T, Cho S, Baek H, Yang XL, et al. Analysis of tumor metabolism reveals mitochondrial glucose oxidation in genetically diverse human glioblastomas in the mouse brain in vivo. Cell Metab. 2012;15:827-37.

8. Kamphorst JJ, Cross JR, Fan J, de Stanchina E, Mathew R, White EP, et al. Hypoxic and Ras-transformed cells support growth by scavenging unsaturated fatty acids from lysophospholipids. Proc Natl Acad Sci U S A. 2013;110:8882-7

9. Metallo CM, Gameiro PA, Bell EL, Mattaini KR, Yang J, Hiller K, et al. Reductive glutamine metabolism by IDH1 mediates lipogenesis under hypoxia. Nature. 2012;481:380-4.

10. Hatzivassiliou G, Zhao F, Bauer DE, Andreadis C, Shaw AN, Dhanak D, et al. ATP citrate lyase inhibition can suppress tumor cell growth. Cancer Cell. 2005:8:311-21.

11. Gaglio D, Metallo CM, Gameiro PA, Hiller K, Danna LS, Balestrieri C, et al. Oncogenic K-Ras decouples glucose and glutamine metabolism to support cancer cell growth. Mol Syst Biol. 2011;7:523.

12. Vizan P, Boros LG, Figueras A, Capella G, Mangues R, Bassilian S, et al. K-ras codon-specific mutations produce distinctive metabolic phenotypes in NIH3T3 mice [corrected] fibroblasts. Cancer Res. 2005;65:5512-5.

13. Bauer DE, Hatzivassiliou G, Zhao F, Andreadis C, Thompson CB. ATP citrate lyase is an important component of cell growth and transformation. Oncogene. 2005;24:6314-22.

14. Porstmann T, Santos CR, Griffiths B, Cully M, Wu M, Leevers S, et al. SREBP activity is regulated by mTORC1 and contributes to Akt-dependent cell growth. Cell Metab. 2008;8:224-36.

15. Griffiths B, Lewis CA, Bensaad K, Ros S, Zhang Q, Ferber EC, et al. Sterol regulatory element binding protein-dependent regulation of lipid synthesis supports cell survival and tumor growth. Cancer Metab. 2013;1:3.

16. Williams KJ, Argus JP, Zhu Y, Wilks MQ, Marbois BN, York AG, et al. An essential requirement for the SCAP/SREBP signaling axis to protect cancer cells from lipotoxicity. Cancer Res. 2013;73:2850-62.

17. Csibi A, Fendt SM, Li C, Poulogiannis G, Choo AY, Chapski DJ, et al. The mTORC1 pathway stimulates glutamine metabolism and cell proliferation by repressing SIRT4. Cell. 2013;153:840-54.

18. Duvel K, Yecies $J$, Menon S, Raman P, Lipovsky Al, Souza AL, et al. Activation of a metabolic gene regulatory network downstream of mTOR complex 1. Mol Cell. 2010;39:171-83.

19. Grassian AR, Metallo CM, Coloff JL, Stephanopoulos G, Brugge JS. Erk regulation of pyruvate dehydrogenase flux through PDK4 modulates cell proliferation. Genes Dev. 2011;25:1716-33. 
20. Kim JW, Tchernyshyov I, Semenza GL, Dang CV. HIF-1-mediated expression of pyruvate dehydrogenase kinase: a metabolic switch required for cellular adaptation to hypoxia. Cell Metab. 2006;3:177-85.

21. Papandreou I, Cairns RA, Fontana L, Lim AL, Denko NC. HIF-1 mediates adaptation to hypoxia by actively downregulating mitochondrial oxygen consumption. Cell Metab. 2006:3:187-97.

22. Semenza GL. HIF-1: upstream and downstream of cancer metabolism. Curr Opin Genet Dev. 2010;20:51-6.

23. Bonnet S, Archer SL, Allalunis-Turner J, Haromy A, Beaulieu C, Thompson R, et al. A mitochondria-K+ channel axis is suppressed in cancer and its normalization promotes apoptosis and inhibits cancer growth. Cancer Cell. 2007;11:37-51

24. Gameiro PA, Yang J, Metelo AM, Perez-Carro R, Baker R, Wang Z, et al. In vivo HIF-mediated reductive carboxylation is regulated by citrate levels and sensitizes VHL-deficient cells to glutamine deprivation. Cell Metab. 2013;17:372-85.

25. Wise DR, Ward PS, Shay JE, Cross JR, Gruber JJ, Sachdeva UM, et al. Hypoxia promotes isocitrate dehydrogenase-dependent carboxylation of alphaketoglutarate to citrate to support cell growth and viability. Proc Natl Acad Sci U S A. 2011;108:19611-6.

26. Zimmer M, Doucette D, Siddiqui N, lliopoulos O. Inhibition of hypoxiainducible factor is sufficient for growth suppression of $\mathrm{VHL}-/-$ tumors. Mol Cancer Res. 2004;2:89-95.

27. Kaplon J, Zheng L, Meissl K, Chaneton B, Selivanov VA, Mackay G, et al. A key role for mitochondrial gatekeeper pyruvate dehydrogenase in oncogene-induced senescence. Nature. 2013;498:109-12.

28. Schell JC, Olson KA, Jiang L, Hawkins AJ, Van Vranken JG, Xie J, et al. A role for the mitochondrial pyruvate carrier as a repressor of the Warburg effect and colon cancer cell growth. Mol Cell. 2014;56:400-13.

29. Vacanti NM, Divakaruni AS, Green CR, Parker SJ, Henry RR, Ciaraldi TP, et al. Regulation of substrate utilization by the mitochondrial pyruvate carrier. Mol Cell. 2014;56:425-35.

30. Yang C, Ko B, Hensley CT, Jiang L, Wasti AT, Kim J, et al. Glutamine oxidation maintains the TCA cycle and cell survival during impaired mitochondrial pyruvate transport. Mol Cell. 2014;56:414-24.

31. Comerford SA, Huang Z, Du X, Wang Y, Cai L, Witkiewicz AK, et al. Acetate dependence of tumors. Cell. 2014;159:1591-602.

32. Mashimo T, Pichumani K, Vemireddy V, Hatanpaa KJ, Singh DK, Sirasanagandla $\mathrm{S}$, et al. Acetate is a bioenergetic substrate for human glioblastoma and brain metastases. Cell. 2014;159:1603-14.

33. Young RM, Ackerman D, Quinn ZL, Mancuso A, Gruber M, Liu L, et al. Dysregulated mTORC1 renders cells critically dependent on desaturated lipids for survival under tumor-like stress. Genes Dev. 2013;27:1115-31.

34. Commisso C, Davidson SM, Soydaner-Azeloglu RG, Parker SJ, Kamphorst JJ, Hackett $\mathrm{S}$, et al. Macropinocytosis of protein is an amino acid supply route in Ras-transformed cells. Nature. 2013;497:633-7.

35. Weinberg F, Hamanaka R, Wheaton WW, Weinberg S, Joseph J, Lopez M, et al. Mitochondrial metabolism and ROS generation are essential for Kras-mediated tumorigenicity. Proc Natl Acad Sci U S A. 2010;107:8788-93.

36. Yang C, Sudderth J, Dang T, Bachoo RM, McDonald JG, DeBerardinis RJ. Glioblastoma cells require glutamate dehydrogenase to survive impairments of glucose metabolism or Akt signaling. Cancer Res. 2009;69:7986-93.

37. Gao P, Tchernyshyov I, Chang TC, Lee YS, Kita K, Ochi T, et al. c-Myc suppression of miR-23a/b enhances mitochondrial glutaminase expression and glutamine metabolism. Nature. 2009;458:762-5.

38. Le A, Lane AN, Hamaker M, Bose S, Gouw A, Barbi J, et al. Glucose-independent glutamine metabolism via TCA cycling for proliferation and survival in B cells. Cell Metab. 2012;15:110-21.

39. Seltzer MJ, Bennett BD, Joshi AD, Gao P, Thomas AG, Ferraris DV, et al. Inhibition of glutaminase preferentially slows growth of glioma cells with mutant IDH1. Cancer Res. 2010;70:8981-7.

40. Wang JB, Erickson JW, Fuji R, Ramachandran S, Gao P, Dinavahi R, et al. Targeting mitochondrial glutaminase activity inhibits oncogenic transformation. Cancer Cell. 2010;18:207-19.

41. Liu W, Le A, Hancock C, Lane AN, Dang CV, Fan TW, et al. Reprogramming of proline and glutamine metabolism contributes to the proliferative and metabolic responses regulated by oncogenic transcription factor c-MYC. Proc Natl Acad Sci U S A. 2012;109:8983-8

42. Scott DA, Richardson AD, Filipp FV, Knutzen CA, Chiang GG, Ronai ZA, et al. Comparative metabolic flux profiling of melanoma cell lines: beyond the Warburg effect. J Biol Chem. 2011;286:42626-34.
43. Son J, Lyssiotis CA, Ying H, Wang X, Hua S, Ligorio M, et al. Glutamine supports pancreatic cancer growth through a KRAS-regulated metabolic pathway. Nature. 2013;496:101-5.

44. DeBerardinis RJ, Mancuso A, Daikhin E, Nissim I, Yudkoff M, Wehrli S, et al. Beyond aerobic glycolysis: transformed cells can engage in glutamine metabolism that exceeds the requirement for protein and nucleotide synthesis. Proc Natl Acad Sci U S A. 2007;104:19345-50.

45. Grassian AR, Parker SJ, Davidson SM, Divakaruni AS, Green CR, Zhang X, et al. IDH1 mutations alter citric acid cycle metabolism and increase dependence on oxidative mitochondrial metabolism. Cancer Res. 2014;74:3317-31.

46. Diehn M, Cho RW, Lobo NA, Kalisky T, Dorie MJ, Kulp AN, et al. Association of reactive oxygen species levels and radioresistance in cancer stem cells. Nature. 2009:458:780-3.

47. Jiang P, Du W, Mancuso A, Wellen KE, Yang X. Reciprocal regulation of p53 and malic enzymes modulates metabolism and senescence. Nature. 2013;493:689-93.

48. Mullen AR, Wheaton WW, Jin ES, Chen PH, Sullivan LB, Cheng T, et al. Reductive carboxylation supports growth in tumour cells with defective mitochondria. Nature. 2012;481:385-8.

49. Fendt SM, Bell EL, Keibler MA, Olenchock BA, Mayers JR, Wasylenko TM, et al. Reductive glutamine metabolism is a function of the alphaketoglutarate to citrate ratio in cells. Nat Commun. 2013;4:2236.

50. Gameiro PA, Laviolette LA, Kelleher JK, Iliopoulos O, Stephanopoulos G. Cofactor balance by nicotinamide nucleotide transhydrogenase (NNT) coordinates reductive carboxylation and glucose catabolism in the tricarboxylic acid (TCA) cycle. J Biol Chem. 2013;288:12967-77.

51. Fan J, Kamphorst JJ, Mathew R, Chung MK, White E, Shlomi T, et al. Glutamine-driven oxidative phosphorylation is a major ATP source in transformed mammalian cells in both normoxia and hypoxia. Mol Syst Biol. 2013;9:712.

52. Kung HN, Marks JR, Chi JT. Glutamine synthetase is a genetic determinant of cell type-specific glutamine independence in breast epithelia. PLoS Genet. 2011;7:e1002229.

53. De Ingeniis J, Ratnikov B, Richardson AD, Scott DA, Aza-Blanc P, De SK, et al. Functional specialization in proline biosynthesis of melanoma. PLoS One. 2012;7:e45190.

54. Nilsson R, Jain M, Madhusudhan N, Sheppard NG, Strittmatter L, Kampf C, et al. Metabolic enzyme expression highlights a key role for MTHFD2 and the mitochondrial folate pathway in cancer. Nat Commun. 2014;5:3128.

55. Hagedorn $\mathrm{CH}$, Phang JM. Transfer of reducing equivalents into mitochondria by the interconversions of proline and delta 1-pyrroline-5-carboxylate. Arch Biochem Biophys. 1983;225:95-101.

56. Liu Y, Borchert GL, Donald SP, Diwan BA, Anver M, Phang JM. Proline oxidase functions as a mitochondrial tumor suppressor in human cancers. Cancer Res. 2009;69:6414-22.

57. Liu W, Zabirnyk O, Wang H, Shiao YH, Nickerson ML, Khalil S, et al. miR-23b targets proline oxidase, a novel tumor suppressor protein in renal cancer. Oncogene. 2010;29:4914-24.

58. Ying H, Kimmelman AC, Lyssiotis CA, Hua S, Chu GC, Fletcher-Sananikone E, et al. Oncogenic Kras maintains pancreatic tumors through regulation of anabolic glucose metabolism. Cell. 2012;149:656-70.

59. Viale A, Pettazzoni P, Lyssiotis CA, Ying H, Sanchez N, Marchesini M, et al. Oncogene ablation-resistant pancreatic cancer cells depend on mitochondrial function. Nature. 2014;514:628-32.

60. Zhang J, Fan J, Venneti S, Cross JR, Takagi T, Bhinder B, et al. Asparagine plays a critical role in regulating cellular adaptation to glutamine depletion. Mol Cell. 2014;56:205-18.

61. Gutierrez JA, Pan YX, Koroniak L, Hiratake J, Kilberg MS, Richards NG. An inhibitor of human asparagine synthetase suppresses proliferation of an L-asparaginase-resistant leukemia cell line. Chem Biol. 2006;13:1339-47.

62. Richards NG, Kilberg MS. Asparagine synthetase chemotherapy. Annu Rev Biochem. 2006;75:629-54

63. Filipp FV, Ratnikov B, De Ingeniis J, Smith JW, Osterman AL, Scott DA. Glutamine-fueled mitochondrial metabolism is decoupled from glycolysis in melanoma. Pigment Cell Melanoma Res. 2012;25:732-9.

64. Holm E, Hagmuller E, Staedt U, Schlickeiser G, Gunther HJ, Leweling H, et al. Substrate balances across colonic carcinomas in humans. Cancer Res. 1995;55:1373-8.

65. Hiller K, Metallo CM, Kelleher JK, Stephanopoulos G. Nontargeted elucidation of metabolic pathways using stable-isotope tracers and mass spectrometry. Anal Chem. 2010;82:6621-8. 
66. Tonjes M, Barbus S, Park YJ, Wang W, Schlotter M, Lindroth AM, et al. BCAT1 promotes cell proliferation through amino acid catabolism in gliomas carrying wild-type IDH1. Nat Med. 2013;19:901-8.

67. Mullen AR, Hu Z, Shi X, Jiang L, Boroughs LK, Kovacs Z, et al. Oxidation of alpha-ketoglutarate is required for reductive carboxylation in cancer cells with mitochondrial defects. Cell reports. 2014;7:1679-90.

68. He T, Haapa-Paananen S, Kaminskyy VO, Kohonen P, Fey V, Zhivotovsky B, et al. Inhibition of the mitochondrial pyrimidine biosynthesis enzyme dihydroorotate dehydrogenase by doxorubicin and brequinar sensitizes cancer cells to TRAlL-induced apoptosis. Oncogene. 2014;33:3538-49.

69. Hu J, Locasale JW, Bielas JH, O'Sullivan J, Sheahan K, Cantley LC, et al. Heterogeneity of tumor-induced gene expression changes in the human metabolic network. Nat Biotechnol. 2013:31:522-9.

70. White RM, Cech J, Ratanasirintrawoot S, Lin CY, Rahl PB, Burke CJ, et al. DHODH modulates transcriptional elongation in the neural crest and melanoma. Nature. 2011;471:518-22.

71. Zhai H, Song B, Xu X, Zhu W, Ju J. Inhibition of autophagy and tumor growth in colon cancer by miR-502. Oncogene. 2013;32:1570-9.

72. Khutornenko AA, Roudko W, Chernyak BV, Vartapetian AB, Chumakov PM, Evstafieva AG. Pyrimidine biosynthesis links mitochondrial respiration to the p53 pathway. Proc Natl Acad Sci U S A. 2010;107:12828-33.

73. Tibbetts AS, Appling DR. Compartmentalization of mammalian folate-mediated one-carbon metabolism. Annu Rev Nutr. 2010;30:57-81.

74. Lewis CA, Parker SJ, Fiske BP, McCloskey D, Gui DY, Green CR, et al. Tracing compartmentalized NADPH metabolism in the cytosol and mitochondria of mammalian cells. Mol Cell. 2014:55:253-63.

75. Anderson DD, Quintero CM, Stover PJ. Identification of a de novo thymidylate biosynthesis pathway in mammalian mitochondria. Proc Natl Acad Sci U S A. 2011;108:15163-8.

76. Ye J, Fan J, Venneti S, Wan YW, Pawel BR, Zhang J, et al. Serine catabolism regulates mitochondrial redox control during hypoxia. Cancer discovery. 2014:4:1406-17.

77. Fan J, Ye J, Kamphorst JJ, Shlomi T, Thompson CB, Rabinowitz JD. Quantitative flux analysis reveals folate-dependent NADPH production. Nature. 2014:510:298-302.

78. Mitsuishi Y, Taguchi K, Kawatani Y, Shibata T, Nukiwa T, Aburatani $H$, et al. Nrf2 redirects glucose and glutamine into anabolic pathways in metabolic reprogramming. Cancer Cell. 2012;22:66-79.

79. Wahrheit J, Niklas J, Heinzle E. Metabolic control at the cytosol-mitochondria interface in different growth phases of CHO cells. Metab Eng. 2014;23:9-21.

80. Evans JM, Donnelly LA, Emslie-Smith AM, Alessi DR, Morris AD. Metformin and reduced risk of cancer in diabetic patients. BMJ. 2005;330:1304-5.

81. Shackelford DB, Abt E, Gerken L, Vasquez DS, Seki A, Leblanc M, et al. LKB1 inactivation dictates therapeutic response of non-small cell lung cancer to the metabolism drug phenformin. Cancer Cell. 2013;23:143-58.

82. Andrzejewski S, Gravel SP, Pollak M, St-Pierre J. Metformin directly acts on mitochondria to alter cellular bioenergetics. Cancer Metab. 2014;2:12.

83. Wheaton WW, Weinberg SE, Hamanaka RB, Soberanes S, Sullivan LB, Anso E, et al. Metformin inhibits mitochondrial complex I of cancer cells to reduce tumorigenesis. eLife. 2014;3:e02242.

84. Hiller K, Metallo CM. Profiling metabolic networks to study cancer metabolism. Curr Opin Biotechnol. 2013;24:60-8.

\section{Submit your next manuscript to BioMed Central and take full advantage of:}

- Convenient online submission

- Thorough peer review

- No space constraints or color figure charges

- Immediate publication on acceptance

- Inclusion in PubMed, CAS, Scopus and Google Scholar

- Research which is freely available for redistribution

Submit your manuscript at www.biomedcentral.com/submit 